\title{
Alinhamento Operacional de Sistemas de Informação: uma alternativa para a melhoria dos serviços prestados pelo setor hoteleiro
}

\section{Alineación Operacional de los Sistemas de Información: una Alternativa para Mejora de la Calidad de los Servicios de la Industria Hotelera}

\section{Information System Operational Alignment: an Alternative Towards Service Quality Improvement in the Hospitality Industry}

Larissa Medianeira Bolzan ${ }^{1}$

Flavio Issao Kubota ${ }^{2}$

Mauri Leodir Löbler ${ }^{3}$

\section{Resumo}

Desde o Século XX, uma transformação foi iniciada por meio de duas novas técnicas da era da informação: a tecnologia da informação (TI) e o redesenho de processos de negócio. Considerando esse contexto, buscando tornar tangível a relação entre processos empresariais e $\mathrm{TI}$, este trabalho tem como objetivo verificar esse relacionamento na indústria hoteleira, a qual está em evidência devido aos megaeventos que o Brasil receberá nos próximos anos. Para isso, buscou-se: mapear todos os processos empresariais da organização unidade de análise; analisar o suporte oferecido aos processos pela tecnologia de informação adotada; verificar o alinhamento entre os processos empresariais e o sistema de informação (SI) adotado e; verificar a satisfação dos usuários com o SI utilizado. O estudo tem caráter qualitativo e natureza descritiva, sendo o método de investigação o estudo de caso único, em um hotel da região central do Estado do Rio Grande do Sul (RS). Para a coleta de dados e informações, utilizou-se da observação não participante e, por fim, aplicou-se uma pesquisa de satisfação com os colaboradores usuários do SI. Como resultados, obteve-se o mapeamento dos processos empresariais da organização, suas inter-relações e o SI adotado. Concluiu-se que os processos estão alinhados com a TI adotada, uma vez que esta é capaz de oferecer suporte àqueles. Ainda, foi constatado que os usuários estão satisfeitos com o SI implantado e entendem que esse sistema é importante no funcionamento da organização.

Palavras-chave: Hotéis; Processos Empresariais; Sistemas de Informação.

\footnotetext{
${ }^{1}$ Doutoranda em Administração pela Universidade Federal do Rio Grande do Sul (UFRGS). Mestre e Graduada em Administração pela Universidade Federal de Santa Maria (UFSM). Brasil. E-mail: larissambolzan@gmail.com

${ }^{2}$ Doutorando em Engenharia de Produção pela Universidade Federal de Santa Catarina (UFSC). Mestre em Engenharia de Produção e Graduado em Engenharia Mecânica pela UFSM. Brasil. E-mail: flavioissao.kubota@gmail.com

${ }^{3}$ Doutor em Administração pela UFRGS. Mestre em Engenharia da Produção e Graduado em Administração pela UFSM. Professor do Programa de Pós-Graduação em Administração da UFSM. Brasil. E-mail: mllobler@hotmail.com
} 


\title{
Resumen
}

Desde principios del siglo XX, una transformación comenzó con dos nuevas técnicas de la era de la información: tecnología de información (TI) y el rediseño de procesos de negocio. Teniendo en cuenta este contexto, tratando de hacer tangible la relación entre los procesos de negocio y de TI, este artículo tiene por objetivo comprobar esta relación en la industria hotelera, que está em pruebas debido a grandes eventos que Brasil recibirá en los próximo años. Para eso, hemos tratado de: asignar todos los procesos de negocio de la organización unidad de análisis; analizar el apoyo prestado, a los procesos, pela tecnología de información adoptado; verificar la alineación entre los processos de negocio y el sistema de información (SI) adoptado y; comprobar la satisfación de los usuarios con el SI utilizado. El estudio es cualitativo y descriptivo y el método de investigación es un estudio de caso único en un hotel de la región central del Rio Grande del Sur (RS). Para la colección de datos y información, se utilizó la observación no participante y, finalmente, aplicar una búsqueda de satisfación con los usuarios de SI. Como resultados, se obtuvo el mapa de procesos de negocio de la compañía, las interrelaciones entre ellos y el SI adoptado. Se encontró que los procesos están alineados con TI utilizado, ya que es capaz de soportar aquellos. Además, se encontró que los usuarios están satisfechos con el SI implantado y comprenden que el mismo es importante para el funcionamiento de la organización.

Palabras clave: Hoteles; Procesos de Negocio; Sistemas de Información.

\begin{abstract}
Since the twentieth century, a transformation has begun through two new techniques from information age: information technology (IT) and business process redesign. Considering this context, aiming to make the relationship between business process and IT tangible, this paper verifies this relationship in the hospitality industry, which is in evidence due to mega events that Brazil will receive in the next years. To conduct this research, we mapped al business processes from the case studied; analyze the support offered by the adopted information technology (IT) to the processes; verify the alignment between business processes and information system (IS) adopted and; verify users satisfaction with the utilized IS. The study is qualitative and descriptive, and the investigation method is a single case study in a hotel located in the central region of Rio Grande do Sul (RS). For the data and information collection, we used the non-participant observation and lastly, we applied a satisfaction survey with IS users. As results, we obtained the business processes mapping from the organization, the interrelationships among them and the IS adopted. We concluded that processes are aligned with IT used, since IT support the business processes. Also, we found that IS users are satisfied with the system adopted and understand that it is important for the organization performance.
\end{abstract}

Keywords: Hotels; Business Processes; Information Systems. 


\section{Introdução}

Recentemente, é possível observar um grande interesse da esfera pública, privada e também da população brasileira em atrair e presenciar eventos esportivos. Isso se justifica pela oportunidade de gerar recursos para a localidade, não apenas àqueles recursos originados pela realização do evento, mas também pela atração de turistas e pela visibilidade dada ao local que o sedia. Essa, encarada como uma conveniência para atrair a atenção de investidores externos (HORNE, 2007; SEIXAS; LOPES, 2012). Para Seixas e Lopes (2012), a promoção de megaeventos esportivos tem sido uma estratégia de diversos países visando investimentos internacionais.

O Brasil sediará dois megaeventos esportivos nos próximos anos, a Copa do Mundo FIFA 2014 e os Jogos Olímpicos de 2016. Seixas e Lopes (2012) afirmam que é inegável a visibilidade que o Brasil terá com a promoção dos megaeventos esportivos supracitados. Os benefícios econômicos destes eventos retratam um argumento utilizado para justificar o esforço e o investimento público para sediar tais acontecimentos (OHMANN et al., 2006; CARVALHO; ALVES, 2009; DOMINGUES et al., 2010; MONZONI NETO et al., 2011; NEVES et al., 2011; SANTOS, 2011; SEIXAS; LOPES, 2012). Nesse contexto, a participação do setor hoteleiro nos debates sobre as futuras ações do Ministério do Turismo é importante para a preparação da cadeia produtiva do turismo (BRASIL, 2012).

Para promover os eventos em questão, é necessário avaliar uma demanda de longo prazo de residentes locais e turistas, considerando lucros para iniciativa privada, mas também ponderando a renda e o trabalho adicional, que produzem maior receita proveniente de impostos para o orçamento público, gerando possibilidades de financiamentos de novos projetos (OHMANN et al., 2006; DOMINGUES et al., 2010). De acordo com Horne (2007), existem três fatores que levam ao crescimento efetivo da sociedade a partir de megaeventos esportivos: o desenvolvimento de novas tecnologias de comunicação em massa, o interesse das grandes corporações em patrocinar tais eventos e a visão dos megaventos como oportunidade para venda, divulgação, promoção de produtos de grandes corporações.

No âmbito organizacional, preocupa-se com os serviços e produtos oferecidos aos clientes. Também deve haver análise e planejamento a respeito da demanda por produtos e serviços. 
Ao iniciar a década de 1990, duas novas ferramentas da era da informação iniciaram uma transformação dentro das empresas (DAVENPORT; SHORT, 1990): a Tecnologia da Informação (TI), que se trata dos recursos oferecidos pelos computadores, aplicativos de software, hardware e telecomunicações, e o redesenho dos processos de negócio. Atualmente, as organizações utilizam tecnologias da informação para a inovação organizacional na geração de ideias, experimentação, comercialização e difusão e implementação de melhorias (LINDIC et al., 2011). Dessa forma, Sheth et al. (2002) afirmam que a TI tem assumido um papel estratégico em diversos setores.

Quanto ao setor hoteleiro, especificamente, destaca-se a importância de análises aprofundadas, pois essas empresas têm papel fundamental quanto à recepção de turistas durante os megaeventos esportivos sediados no Brasil, e também quanto à impressão cunhada nesses investidores por meio de serviços oferecidos a eles. Além disso, a hotelaria também pode surgir como um elemento importante em estratégias ampliadas de desenvolvimento econômico (ESTEVÃO et al., 2009), principalmente considerando os países em desenvolvimento, onde a taxa de crescimento do turismo internacional foi superior a oito por cento (8\%), o que é superior aos países desenvolvidos onde o crescimento foi, em média, de $5 \%$, evidenciando, dessa forma, que as economias emergentes estão enfrentando um rápido crescimento no turismo (JAUHARI; RISHI, 2012).

Considerando os dados do Instituto Brasileiro de Turismo (EMBRATUR), em 2011, com a economia em um momento positivo e o planejamento para a realização da Copa 2014 em território brasileiro, o país apresenta-se em estágio de transformação para um significativo aumento no número de turistas estrangeiros, as informações provenientes do Ministério do Turismo apontam um aumento de 5 milhões para 8 milhões até 2014 (BRASIL, 2011). Panorama esse também corroborado por Teixeira (2011), o autor relata que no Brasil, o desenvolvimento do setor turístico tem sido estimulado pelos responsáveis por políticas econômicas porque contribui não apenas para atrair capital externo, mas também para a geração de novos empregos diretos e indiretos.

Para o anfitrião da Copa 2014 deixar uma boa imagem, o Governo, em parceria com a EMBRATUR, criou o programa Bem Receber Copa. O eixo principal de atuação desse programa diz respeito a qualificar o setor de turismo, focado em pessoas, empresas e destinos 
(BRASIL, 2011). Nesse sentido, a TI e o gerenciamento de processos são vistos como fortes aliados na criação de novas estratégias de negócio, de novas estruturas organizacionais, de novas formas de relacionamento entre empresas e entre empresas e seus consumidores. Uma vez que o gerenciamento de processos empresariais e o alinhamento desses com a tecnologia de informação, a qual fornece suporte aos processos, são capazes de permitir um melhor funcionamento da empresa com foco na sequência de atividades que levem os produtos e serviços até o cliente (LAURINDO; ROTONDARO, 2006). Para Ramos e Rodrigues (2006), o desenvolvimento da tecnologia da informação tem criado um número significativo de ferramentas para a integração dos processos hoteleiros. Tal afirmação também é justificada pelo estudo de Sheth et al. (2002), o qual afirma que a TI está cada vez mais assumindo um papel estratégico em diversos setores.

Para Davenport e Short (1990), a importância da conexão entre TI e redesenho de processos empresariais é bastante conhecida entre os engenheiros, embora essas ferramentas ainda estejam sendo usadas de forma separada. Os mesmos autores conceituam TI como a representação de processos por meio de softwares de gestão. Nesse sentido, Nolan (1979) afirma que a integração entre TI e Gestão por Processos nas atividades empresariais é o estágio final da evolução do uso de tecnologias de informação.

$\mathrm{Na}$ busca por tornar tangível a relação entre processos empresariais e tecnologia da informação (TI), este artigo tem como objetivo explorar o relacionamento entre os processos empresariais e a TI adotada, tomando por direção a teoria existente a respeito do tema, por meio de um estudo de caso único em um hotel da região central do Estado do Rio Grande do Sul (RS). Para tanto, desenvolveu-se os seguintes objetivos específicos que balizaram a realização deste trabalho: (i) mapear todos os processos da empresa que serviu com unidade de análise; (ii) analisar o suporte oferecido pela Tecnologia de Informação adotado pela unidade de análise; (iii) verificar o alinhamento entre os processos empresariais e o sistema de informação adotado, e; (iv) verificar a satisfação dos usuários com o sistema de informação adotado adotada.

Diante do exposto, este estudo justifica-se por possibilitar uma maior compreensão dos fatores que facilitam e/ou limitam o alinhamento entre processos empresariais e a Tecnologia da informação adotada, além de contribuir com a teorização sobre o tema, uma vez que há 
poucos estudos nesse campo. Para atingir o objetivo proposto, o conceito de Tecnologia da Informação será mantido como ferramenta de gestão que contempla, além dos recursos tecnológicos de hardware e software, o Sistema de Informação (SI) adotado (WARD; PEPPARD, 2002; O'BRIEN, 2010). Como conceito de SI, utilizou-se o proposto por Turban et al. (2007), que entende um sistema de informação como um sistema baseado em computadores que coleta, processa, armazena, analisa e dissemina informações com o objetivo de gerar conhecimento para resolver problemas organizacionais.

O trabalho está estruturado da seguinte maneira, posterior a esta introdução: na seção 2, encontra-se o referencial que forneceu a fundamentação teórica necessária para o estudo. Em seguida (seção 3), apresentam-se os procedimentos metodológicos que viabilizaram a realização do trabalho. Por fim, nas seções 4 e 5, respectivamente, são descritos os resultados obtidos a partir das observações realizadas, bem como pesquisa de satisfação com os usuários de SI da unidade de análise, e as conclusões encontradas a partir do trabalho, identificando o alinhamento entre os constructos considerados (processos empresariais e SI), verificando a convergência do estudo com a literatura vigente no tema e as limitações e oportunidades futuras emergidas após o término desta investigação.

\section{Referencial Teórico}

\subsection{Processos empresariais e o redesenho de processos organizacionais}

Processos são definidos como uma série sistemática de ações direcionadas para a consecução de uma meta (JURAN, 1995). Outra definição de processo é a de Campos (1999), que salienta a ideia de operações sucessivas e/ou paralelas que proporcionam um resultado, geralmente como parte de um ciclo global de produção, de um produto ou de um serviço. Para Laurindo e Rotondaro (2006), processo é somente a forma como é desenvolvida uma atividade.

Segundo Davenport e Short (1990), a classificação dos processos depende de três grandes dimensões: tipo de organização, tipo de objetos manipulados e tipo de atividade realizada durante o processo. Dentro desses conjuntos estão os tipos de processos. Quanto à dimensão organização, tem-se que os processos podem ser interorganizacionais, interfuncionais e departamentais; no que tange a dimensão objetos, tem-se os tipos: manufatura e serviços, e; 
no que se refere a atividades tem-se os tipos: operacional e de gerenciamento. A Figura 1 mostra as relações entre as dimensões e tipos de processos.

\begin{tabular}{|c|l|}
\hline Dimensões dos Processos & \multicolumn{1}{c|}{ Tipos de Processos } \\
\hline \multirow{2}{*}{ Organizações } & $>$ Interorganizacional; \\
& $>$ Interfuncional; \\
& $>$ Departamental. \\
\hline Objetos & $>$ Manufatura; \\
& $>$ Serviços. \\
\hline \multirow{2}{*}{ Tipo de Atividades } & $>$ Operacional; \\
& $>$ Gerenciamento. \\
\hline
\end{tabular}

Figura 1 - Relação entre as dimensões e tipos de processos Fonte: DAVENPORT; SHORT, 1990, pg.15

Coutinho e Chagas (2009) salientam que na administração de serviços, os processos são realizados de forma interativa com o cliente e é por esse motivo que se torna necessário detalhar toda a sequência da operação. Precisa-se entender o funcionamento da parte visível das operações, mas sem esquecer que a parte invisível atua como apoio às operações realizadas.

De acordo com Davenport e Short (1990), se a empresa perceber que há necessidade de reformular seus processos, o redesenho empresarial é desenvolvido em cinco etapas: o desenvolvimento de objetivos dos negócios e dos processos, a identificação dos processos a ser redesenhados, a compreensão dos processos existentes, a identificação do nível de integração da Tecnologia da Informação com cada processo, e, em seguida, o redesenho de cada processo. A Figura 2 mostra as cinco etapas do redesenho de processos empresariais. 


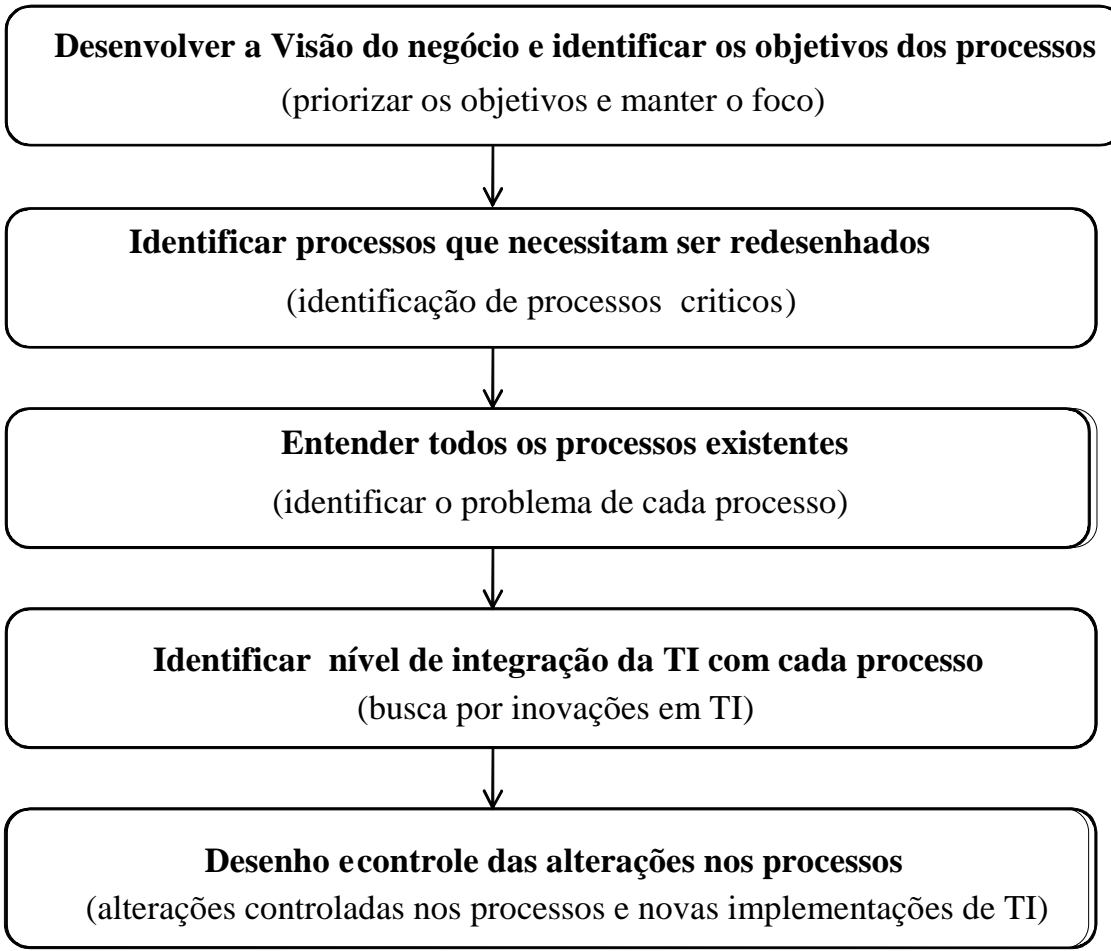

Figura 2 - Os cinco passos do redesenho de processos empresariais (adaptado de Davenport e Short, 1990, pg. 06).

A técnica de otimização de processos, que também pode ser chamada de Business Process Redesign (BPR), consiste em realizar interferências nos processos, provocando alterações planejadas e com resultados controlados, cujo propósito é de observar mudanças e seus respectivos efeitos (JURAN, 1995). O objetivo da aplicação dessa metodologia são atividades com tempo e custo minimizados e com manutenção e confiabilidade (CASTELLI, 2003).

\subsection{Tecnologia da Informação na indústria hoteleira}

Os empreendimentos hoteleiros exercem um importante papel dentro dos negócios relacionados à atividade turística (GOHR; SANTOS, 2010). Com a crescente demanda por informações por parte dos clientes e especialistas do setor, os hotéis têm adotado sistemas baseado em tecnologias de informação para melhorar a eficiência operacional, reduzir custos e aumentar a qualidade dos serviços (VAN HOOF et al., 1996; SIGUAW; ENZ, 1999; CAMISON, 2000; COBANOGLU et al., 2001). 
No entendimento de Turban et al. (2007), os Sistemas de Informação (TI) são recursos integrais de cada área funcional. Para Ward e Peppard (2002) e O'Brien (2010), um Sistema de Informação (SI) é um conjunto organizado de pessoas, hardware, software, redes de comunicação e recursos de coleta, transformação e disseminação de informações em uma organização. Esses componentes, inter-relacionados, transformam recursos de dados em produtos de informação.

O uso da TI facilita às organizações, principalmente às do setor hoteleiro: segmentar seus clientes no momento em que eles preenchem cadastros; aplicar tarifas diferenciadas; fornecer informações instantâneas; personalizar o atendimento na reserva, no check-in, durante a estada, e no check-out (ROSEN; HOWARD, 2000; YELKUR; DACOSTA, 2001; CARVELL; QUAN, 2008). Assim, o cliente satisfeito tende a ampliar sua frequência e, por fim, tornar-se leal à empresa (KANDAMPULLY; SUHARTANTO, 2000).

Assim, com o uso da TI nos negócios, os gestores de hotéis esperam que suas margens de lucro e retornos financeiros cresçam (LAW; JOGARATNAM, 2005). O investimento em TI, desse modo, beneficia o hotel caso permita oferecer aos clientes uma melhor experiência e que a equipe trabalhe de forma mais eficiente para melhor atender aos clientes. Por isso, gestores e empreendedores desejam, além de conhecimentos em idiomas e de hotelaria, de modo geral, obter conhecimentos em informática (WATKINS, 1995; TEIXEIRA, 2011), visando um melhor aproveitamento da TI nos negócios.

De modo geral, a indústria hoteleira é considerada de informações intensas, pois lida com uma quantidade elevada de documentos, recursos e conhecimentos em sua rotina. Davis e Davidson (1991) mencionam que com a TI reformulando a estrutura básica do setor e da sociedade, bem como as necessidades dos clientes por informações mais rápidas e claras, a velocidade da difusão tecnológica em hotéis crescerá consideravelmente. Mas para isso, é necessário que se mantenha interação com os clientes, funcionários devidamente preparados, processos ágeis e flexíveis, bem como qualidade nos serviços, para que a indústria hoteleira tenha maior vantagem competitiva e produtividade (LIMA FILHO et al., 2012).

Esse panorama pode ser potencializado em território brasileiro, uma vez que megaeventos esportivos como as próximas Copa do Mundo (2014) e Olimpíadas (2016) ocorrerão no país. E, conforme Seixas e Lopes (2012), os organizadores de megaeventos esportivos como esses 
estimulam negócios domésticos (restaurantes, hotéis, comércio e prestadores de serviços), o que consequentemente apresenta inúmeros benefícios econômicos que sobressaem aos recursos investidos. Tais efeitos são evidenciados em relatório de impactos econômicos e servem como justificativa para investimentos em infraestrutura e em programas que visam a qualificação de recursos humanos (SANTOS, 2011; SEIXAS; LOPES, 2012).

Além disso, é importante que os hotéis mantenham contatos próximo com a indústria de TI. Esse contato aproximado é necessário para manter os gestores de hotéis atualizados acerca dos desenvolvimentos recentes em TI. Assim, os gestores podem, então, integrar as instalações de TI apropriadas em seus negócios, o que contribui para que os hotéis se mantenham competitivos na indústria, uma vez que as capacidades da TI são limitadas mais pela atitude da gerência dos hotéis, treinamento, habilidades, ambições e situação financeira do que em relação às limitações técnicas de sistemas de software e hardware (LAW; JOGARATNAM, 2005).

\subsection{O alinhamento de TI e o impacto organizacional}

No âmbito empresarial, para Laudon e Laudon (2004, p. 9), os SI correspondem em uma "solução organizacional e administrativa baseada na Tecnologia da Informação para enfrentar um desafio proposto pelo ambiente". Os autores destacam também que para entendê-los completamente é preciso conhecer melhor as organizações, a tecnologia que pode ser utilizada, o ambiente empresarial e os desafios que estas três dimensões podem trazer para as empresas.

Farrell (2005) destaca que a TI pode trazer ganhos importantes se associada a mudanças em processos organizacionais. A trilha para o sucesso de uma organização não está relacionada com o hardware e o software utilizados, ou ainda com as metodologias de desenvolvimento do sistema de informação, mas com o alinhamento da TI adotada, à estratégia, às características e à estrutura organizacional (ROCKART et al., 1996). Segundo Laurindo e Rotondaro (2006), é notável, nas organizações, a dificuldade de alinhamento das tentativas de melhorias com os objetivos e as necessidades das empresas.

Devido às turbulências constantes que o mercado vem sofrendo, as organizações devem mudar seus planos ou readaptá-los sob o risco de se tornarem pouco competitivas. Desta 
forma, são forçadas a constantes mudanças que demandam agilidade e flexibilidade de adaptação dos seus processos. Santos (2006) afirma que é nesse contexto que a TI torna-se imprescindível para dar suporte ao gerenciamento de processos, alcançando, assim, o alinhamento entre processos empresariais e tecnologia da informação.

No que tange a competitividade, Laurindo e Rotondaro (2006) enfatizam que a manutenção desta é obtida entrelaçando dois conceitos: Gestão por Processos e Tecnologia da Informação, assegurando que esses possibilitam as mudanças que uma empresa precisa para se tornar competitiva na acirrada disputa por mercados locais e mundiais.

O êxito do uso de TI nas organizações é efetivo quando sua utilização está alinhada com as estratégias empresariais, com o objetivo e com a utilização eficiente de hardware, software ou metodologias de programação (PORTER; MILLAR, 1999; FERNANDES; ALVES, 1992, apud REZENDE; ABREU, 2003). A utilização estratégica da TI proporciona um conhecimento estratégico essencial para a sobrevivência da organização; por outro lado, a utilização massiva da tecnologia pode não ser suficiente para garantir o sucesso da execução dos processos (BRODBECK; GALLINA, 2007).

O alinhamento pode ser encontrado nas organizações basicamente em dois níveis. O primeiro nível é o de integração operacional, para o qual há a necessidade de planos operacionais de negócio e de TI (SANTOS, 2006). Esse nível é caracterizado pela especificação de requisitos e funções dos SI e do negócio em nível operacional, abrangendo estrutura e processos organizacionais que usam a TI como suporte. Sendo assim, neste nível, a TI é considerada promotora de alinhamento, uma vez que é o ponto de apoio à integração dos negócios por meio de dados, processos, pessoas e redesenho organizacional (HENDERSON; VENKATRAMAN, 1993; SABHERWAL; CHAN, 2001; KAPLAN; NORTON, 2006).

O segundo nível é o Alinhamento Estratégico, que mostra a ideia de integração em um nível mais alto de gestão, onde a integração ocorre por meio da adequação estratégica dos objetivos da TI com as estratégias, objetivos e competências fundamentais do negócio, podendo ocorrer redirecionamento do negócio através da TI (LUFTMAN et al., 1993; LUFTMAN et al, 2004). Apesar de existir pouca literatura sobre alinhamento operacional de TI, pode-se concluir que o alinhamento operacional entre TI e processos empresariais trata da integração entre essas duas 
variáveis. O alinhamento operacional ocorre quando a Tecnologia da informação dá suporte integral aos processos empresariais e os usuários estão adaptados à interface. Uma consequência do alinhamento entre TI e negócio é que, quanto mais um SI está alinhado com os objetivos de negócio da empresa, mais sua importância é percebida (BROADBENT; WEILL, 1993; WEILL; VITALE, 1999).

\section{Procedimentos Metodológicos}

Para a consecução deste trabalho, realizou-se uma pesquisa com abordagem qualitativa, devido ao fato de estudos desse caráter proporcionar maior compreensão acerca de determinadas características estudadas, bem como a possibilidade de gerar informações aprofundadas acerca do assunto (HAIR et al., 2006). Quanto à natureza, foi um estudo exploratório, que conforme Gil (2010), trata-se de um estudo que proporciona uma maior flexibilidade na busca de informações sobre determinado problema, visando torná-lo mais claro, e possibilitando a construção de novas ideias sobre o tema abordado.

Em seguida, definiu-se a empresa a ser utilizada como unidade de análise. A escolha da organização foi do tipo intencional ou seleção racional (BARROS; LEHFELD, 2007), considerando a importância exercida por tal organização na região onde está inserida e em função da forma como é organizada. Desse modo, os critérios de seleção não se restringiram apenas à facilidade de acesso à organização (CAUCHICK MIGUEL, 2010).

Quanto ao método de investigação, a pesquisa caracteriza-se por ser um estudo de caso único, o qual apresenta benefícios quanto ao aprofundamento na investigação, no entanto possui limitações quanto ao grau de generalização dos resultados (CAUCHICK MIGUEL, 2010). Para Gil (2010), o estudo de caso proporciona uma visão global do problema possibilitando a identificação de possíveis fatores que o influenciam ou são por ele influenciados. Cauchick Miguel (2010) complementa afirmando que os principais benefícios da condução de um estudo de caso estão a possibilidade do desenvolvimento de novas teorias e aumentar o conhecimento sobre eventos reais e contemporâneos. Os objetos de análise "processos empresariais" e "Tecnologia da Informação" foram estudados na organização mencionada anteriormente. 
Para a operacionalização deste trabalho, inicialmente foi realizada a observação não participante, que permitiu a visualização do funcionamento da empresa com foco na sequência de atividades que fazem com que os produtos e serviços cheguem ao cliente (LAURINDO; ROTONDARO, 2006). Nesse tipo de observação, o investigador não é parte do processo, apenas atua como espectador atento (RICHARDSON, 1999).

Finalizadas as observações, uma pesquisa de satisfação com todos os colaboradores que trabalham com o sistema de informação foi aplicada, o que permitiu avaliar a perspectiva por parte dos usuários - acerca da TI adotada. Atividade importante para caracterizar o sucesso do sistema de informação e garantir a sua continuação (MAÇADA; BORENSTEIN, 2000). Ainda quanto à pesquisa de satisfação, utilizou-se o instrumento validado por Iivari (2005) para a obtenção das informações necessárias à investigação. Por fim, a Figura 3 apresenta, resumidamente, as etapas realizadas para a execução do presente trabalho. 


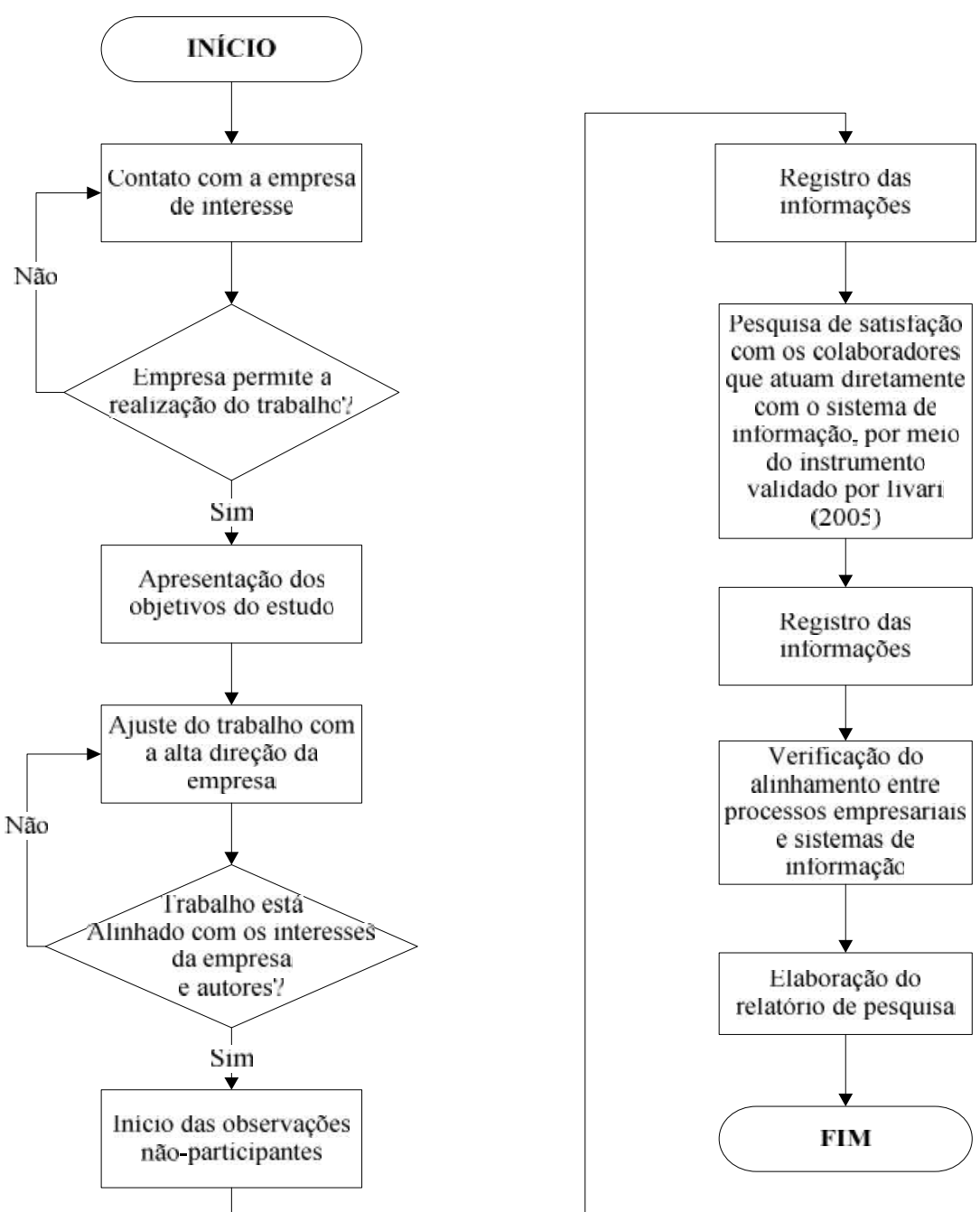

Figura 3 - Etapas da pesquisa.

\section{Resultados e Discussão}

\subsection{Mapeamento de processos empresariais}

A empresa a qual serviu como unidade de análise é composta por seis departamentos: Departamento de Eventos e Convenções, Departamento de Hospedagem, Departamento de Alimentos e Bebidas, Departamento de Compras, Departamento Financeiro e Departamento de Relações Humanas. A investigação iniciou pelo Departamento de Eventos e Convenções, que é responsável por atender clientes e direcioná-los à melhor solução quanto a reservas de salas para realização de eventos, sejam esses particulares ou empresariais. É dividido em duas 
áreas funcionais: Comercial, responsáveis pela venda das salas de eventos e Montagem, responsável pelas alterações de layout das salas para o evento.

Esse departamento é responsável por sete processos: agendamento de evento (A.E.), confecção do mapa semanal (programação) (C.M.S.); confecção do resumo de alimentos e bebidas (C.R.A.B.), que trata de relatórios semanais da necessidade de alimentos e bebidas para os eventos; preparação da sala para evento (P.S.); encerramento de evento (E.E.); agendamento de vernissage (A.V.) - trata-se do controle de datas do aluguel do hall para exposição de quadros de artistas locais e regionais e; processo de candidatura a processo licitatório (P.L.).

Alguns processos do Departamento Eventos e Convenções resultam, também, em geração de demanda para os Departamentos de Hospedagem e de Alimentos e Bebidas, uma vez que alguns participantes de eventos pernoitam - utilizando assim, serviços de hospedagem - e também há eventos que necessitam de alimentação em intervalos pré-definidos - geralmente coffee break e almoço - utilizando serviços do Departamento de Alimentos e Bebidas (A\&B).

O segundo departamento observado foi o Departamento de Hospedagem, responsável por um atendimento de qualidade aos hóspedes, assim como por direcioná-los à melhor solução quanto à estada. Apresenta-se dividido em três áreas funcionais: recepção, com propósito do atendimento aos hóspedes; governança, com o objetivo de proporcionar um ambiente limpo e agradável, e; portaria.

O Departamento de Hospedagem é responsável por oito processos: reserva de apartamento (R.A.); check-in ou walk-in e alocação do hóspede; organização de apartamentos (O.A.); venda dos produtos da vitrine (V.P.V.); fechamento de caixa (F.C.); confecção do relatório de ocupação para o café da manhã (R.C.M.) - por meio da geração de um relatório no SI; processo de controle de lavanderia (P.L.) - quando as roupas dos hóspedes são enviadas à lavanderia, lavadas, recebidas e devolvidas ao hóspede, e; check-out - quando o valor da diária e dos serviços utilizados é informado ao cliente e este faz o pagamento, encerrando a estada.

Em seguida, foi observado o Departamento de Compras, que tem como função suprir a necessidade de insumos dos demais departamentos, bem como dos pontos de venda. Embora 
esse departamento não esteja dividido em áreas funcionais, foi percebida uma divisão informal entre as atividades de controle e compras, que tratam da verificação da necessidade de aquisição e da aquisição de insumos, e as atividades de abastecimento de insumos às demais áreas funcionais da empresa, que tratam da separação, transporte e entrega dos insumos ao departamento solicitante. Os processos de responsabilidade do Departamento de Compras são: compras (CMP); recebimento de mercadorias (R.M.); atendimento de requisições (A.R.); acerto de estoque (A.E.) (esse processo acontece quando são repassados insumos de matriz para filial ou vice-versa) e o processo de devolução de insumo (D.I.) quando um departamento faz pedido errado de insumos e os devolve ao almoxarifado.

Outro departamento observado foi o Departamento de Alimentos e Bebidas, responsável por atender a demanda de alimentação do restaurante do hotel e dos participantes dos eventos. Esse departamento é responsável por sete processos: recebimento de insumos (R.I.) - quando os insumos são entregues a área funcional da cozinha; preparo das refeições (P.R.); montagem do buffet (MNT); controle de estoque (C.E.) - realizado após o café da manhã; atendimento no restaurante (A.R.) - que trata do atendimento aos clientes, e do controle e reposição do buffet. Os processos específicos da área funcional restaurante são o Lançamento de Despesa (L.D.) - no apartamento do hóspede ou geração de nota fiscal - e; recebimento de pagamentos (R.P.).

Por último, foi acompanhada a rotina do Departamento Financeiro, responsável por controlar as entradas e saídas de valores e também por gerar relatórios que dão suporte para decisões de investimento da empresa. Neste departamento, destacam-se os processos de cobrança de valores a receber (P.C.); envio de objetos esquecidos (E.O.E.) - tanto pelos hóspedes quanto pelos participantes dos eventos; o processo de pagamento de contas (P.C.) e; o ajuste do fluxo de caixa (A.F.C.).

A respeito da análise de processos, observaram-se retrabalhos, tais como uso de fichas e do sistema, resultando na permanência de banco de dados idênticos, o que leva a desperdícios de recursos. Destacou-se, por retrabalho, o Departamento de Hospedagem, mais especificamente a área funcional Recepção, uma vez que apresentou retrabalho em muitos de seus processos.

Essa etapa resultou no mapeamento de 33 processos. A análise dos processos da empresa pesquisada permitiu observar que a inter-relação existente entre os processos é forte e que 
muitos processos são complementares. Isso gera forte união entre as áreas funcionais da organização, como já observado por Davenport e Short (1990). A partir do mapeamento, pode-se concluir também que este objeto de análise está de acordo com os objetivos da empresa, com o negócio e com as estratégias traçadas. Assim, pode-se afirmar que está voltado a atender as necessidades e desejos dos clientes, acionistas, parceiros, colaboradores e sociedade.

\subsection{O Sistema de Informação adotado}

A empresa pesquisada utiliza o sistema de informação (SI) como suporte a processos empresariais, alternativa de comunicação e banco de dados. Trata-se de um sistema Enterprise Resource Planning (ERP), pois permite o planejamento e o gerenciamento dos recursos da organização (TURBAN et al., 2007). É denominado Desbravador, adotado na versão 3.0 (DESBRAVADOR, 2012).

O usuário tem acesso ao SI digitando seu login e senha na tela de abertura. O cadastro do usuário é feito pelo gerente do Departamento de Eventos e Convenções que é responsável pelo SI. A empresa adotou seis módulos do sistema, os quais são: Módulo Eventos e Convenções, utilizado pelo Departamento de Eventos e Convenções; Módulo Gerência Hoteleira, utilizado principalmente pelo Departamento de Hospedagem; Módulo Gerência Financeira, utilizado pelo Departamento Financeiro; Módulo Controle de Estoque, utilizado principalmente pelo Departamento de Compras, o Módulo Ponto de Vendas, utilizado pelas áreas funcionais restaurante e $p u b$ e o Tarifador Eletrônico, que controla as ligações realizadas pelo hóspede e lança diretamente no módulo Gerência Hoteleira, utilizado pela área funcional de hospedagem.

Quanto ao Departamento Comercial, foi observado que há necessidade de apoio do sistema em todos os processos realizados. Em seis desses (conforme apresenta a Figura 4) foram observados a utilização do Módulo Eventos e Convenções e, em um específico - processo de encerramento de evento (E.E.) - foi observada a utilização do Módulo Gerência Hoteleira, geralmente utilizado pelo Departamento de Hospedagem.

No que se refere ao Módulo Gerência Hoteleira, observou-se que esse dá suporte a todos os processos do Departamento de Hospedagem. Desde a reserva do apartamento até o processo 
de encerramento da hospedagem. Além de fornecer informações quanto às entradas de caixa relativas a diárias (também representado na Figura 4).

Ligado ao Módulo Gerência Hoteleira tem-se o Tarifador Eletrônico. A esse, é atribuído a função de controlar as ligações telefônicas dos hóspedes. O controle é feito pelos números dos apartamentos, e em caso dos hóspedes utilizarem o telefone para ligações, o custo dessas é tarifado diretamente na conta do apartamento.

O Departamento Financeiro utiliza o Módulo Gerência Financeira, que tem capacidade de dar suporte para a realização das atividades desse departamento. Esse módulo tem acesso a todos os outros módulos devido à responsabilidade de manter atualizado o fluxo de caixa para controle de despesas e estudar possibilidades de novos investimentos.

Durante o acompanhamento do Departamento de A\&B, observou-se pouca utilização do sistema de informação, uma vez que a maioria das suas atividades não necessita desse tipo de suporte. No que se refere à utilização do sistema, são utilizados dois módulos: o Módulo Ponto de Vendas, pela área funcional Restaurante, para atendimento da demanda do restaurante, e; o Módulo Controle de Estoque, para controlar e solicitar insumos para o preparo da alimentação programada.

Quando se trata do Módulo Controle de Estoque, utilizado predominantemente pelo Departamento de Compras, foi observada a necessidade que o sistema de informação supre a respeito da comunicação entre esse departamento e os demais, uma vez que todos os gerentes tem acesso a esse módulo e fazem pedidos de insumos por intermédio desse.

Dessa forma, a análise do SI adotado pela empresa que serviu como objeto de estudo permitiu observar que essa ferramenta é capaz de oferecer suporte necessário aos processos da organização, uma vez que o sistema atende as diversas atividades as quais necessitam do mesmo.

\subsection{Processos empresariais e Sistema de Informação adotado: análise de alinhamento}

No que tange a inter-relação entre os processos empresariais e o sistema de informação adotado foi observado que o SI é capaz de oferecer suporte às necessidades diárias da empresa. Foi percebido também que alguns processos, principalmente aqueles que exigem controle, despenderiam muito mais tempo, recursos e talvez contratações para a empresa, caso 
não existisse o SI. Isso é justificado pela teoria defendida por Laudon e Laudon (2004, p. 426) de que "os sistemas de informação podem facilitar a sobrevivência da empresa". Assim, pode ser identificada existência de uma estreita relação entre os processos empresariais e o SI adotado pela empresa.

A Figura 4 representa a inter-relação do SI adotado pela empresa e seus processos empresariais. Os quadrados com bordas coloridas e não preenchidos com cores representam os processos empresariais e os preenchidos pela mesma cor da borda representam os módulos do SI adotado. Os processos estão definidos por siglas, que são as letras iniciais das palavras que servem de título do processo. 


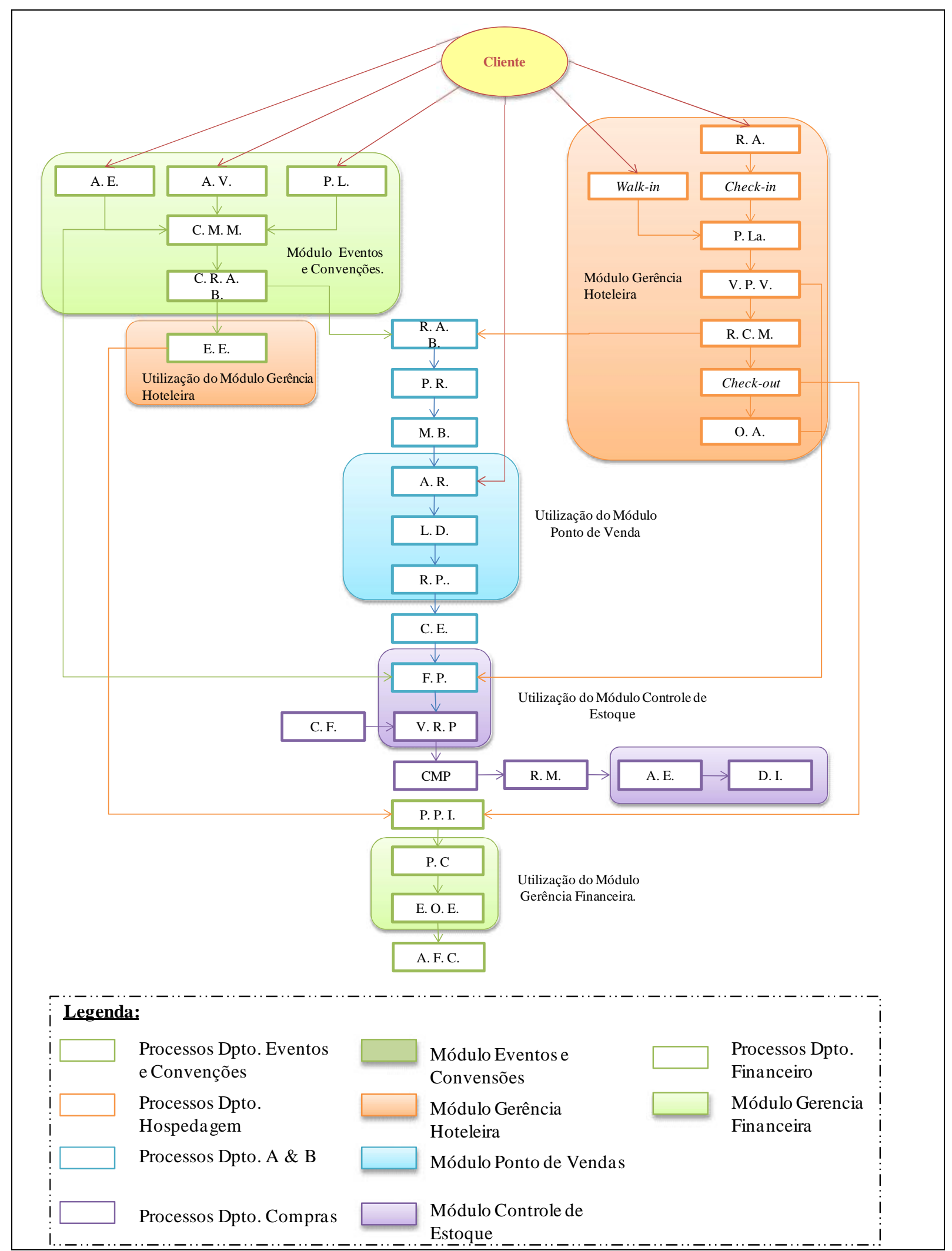

Figura 4 - Inter-relação entre os processos empresariais e o sistema de informação adotado. 
Quanto aos processos do Departamento Eventos e Convenções (representado pela cor verde), foi observado que o Módulo Eventos e Convenções do SI adotado apoia cinco dos seis processos. No que tange o processo de encerramento de evento (E.E.) é concluído no Módulo Gerência Hoteleira.

O Departamento de Hospedagem, representado pela cor laranja, tem o suporte do Módulo Gerência Hoteleira tanto na inserção dos dados quanto na extração de relatórios, que gerados de forma detalhada e segura, objetivam a transparência e a confiabilidade dos processos da organização.

Poucos processos do Departamento de Alimentos e Bebidas necessitam de suporte do sistema de informação, os que necessitam são os ligados ao atendimento do restaurante, pub, room service e controle de insumos. O módulo que oferece suporte a área funcional restaurante, pub e room service é o denominado Ponto de Vendas, e está representado na Figura 4 pela cor azul. A partir da análise, pode-se concluir que os recursos oferecidos pela tecnologia de informação adotada estão alinhados às atividades. Outro módulo essencial para esse departamento é o Módulo Controle de Estoque, que também auxilia os processos do Departamento de Compras.

É importante destacar que a inter-relação entre o Departamento de Compras e o Departamento de Alimentos e Bebidas é forte devido à frequência e a urgência de insumos para preparo do buffet e também nos eventos. Quanto ao Departamento de Compras, este tem seu funcionamento totalmente voltado ao suporte do SI. Os inputs das suas atividades diárias são recebidos através da característica de gerar relatório desse módulo (representado pela área roxa). Salienta-se nesse módulo que, caso ocorra alguma solicitação feita e entregue de maneira errada, o insumo pode ser devolvido, por meio de um recurso do sistema de informação denominado "devolução de itens ao estoque". A presença desse recurso mostra a adequação da tecnologia de informação a necessidade da organização de manter a transparência nos processos.

A respeito dos processos do Departamento Financeiro, tem-se o suporte do Módulo Gerência Financeira. Esse módulo permite comunicação com o sistema bancário, caracterizando um sistema interorganizacional, conforme classificação de Turban et al. (2007), tecnologias que conectam diferentes empresas, e caracterizando esse processo também como 
interorganizacional (DAVENPORT; SHORT, 1990). É por meio dessa facilidade que se controla o pagamento do boleto bancário enviado aos clientes que optam pela modalidade de pagamento faturado.

A inter-relação entre os processos do Departamento Financeiro e Módulo Gerência Financeira mostra claramente a possibilidade de integração entre os módulos do SI adotado, uma vez que para realizar a cobrança aos clientes, o Módulo Gerência Financeira recebe informações dos Departamentos de Hospedagem e de Eventos e Convenções, o que também caracteriza o sistema e o processo como interfuncionais (TURBAN et al., 2007; DAVENPORT; SHORT, 1990).

Com relação ao alinhamento torna-se importante salientar a característica de customização dos módulos. O Módulo Eventos e Convenções, por exemplo, possui a mesma identificação das salas no sistema como na estrutura física, do mesmo modo o Módulo Gerência Hoteleira tem a mesma identificação que os apartamentos possuem. Além disso, permite que o administrador do sistema atualize os valores dos produtos e serviços.

Quanto ao Módulo Gerência Hoteleira, é importante salientar que a organização dos apartamentos oferecida pelo SI adotado, em categorias como "luxo casal", "luxo single", entre outras, é customizada, permitindo adaptação do Sistema de Informação a qualquer realidade ou até mesmo reforma da estrutura física e possível mudança, denotando também em flexibilização, característica importante de um Sistema de Informação.

Importante destacar também a geração de relatórios. O SI gera relatórios de entradas e saídas de recursos financeiros, número de hóspedes diários, semanais e mensais, número de refeições servidas no restaurante e em eventos, valores de insumos repassados a cada centro de custo (departamento), entre outros. Gera também relatórios estatísticos relacionados a receitas e despesas da empresa com atividades de determinado departamento, apontando sazonalidades. A capacidade de gerar essas informações é essencial para o gerenciamento dos processos da empresa, uma vez que não se gerencia aquilo que não se mede (KAPLAN; NORTON, 1997).

Pode-se verificar, ainda, que alguns aspectos no alinhamento entre os processos e o SI adotado convergem com a literatura. Foi percebido que o uso da TI contribui para atividades como o preenchimento de cadastro dos clientes, controle de estoque e finanças, atendimento 
personalizado nas reservas e demais atividades desde o check-in, perpassando pela estada até o check-out dos clientes da empresa (ROSEN; HOWARD, 2000; YELKUR; DACOSTA, 2001; CARVELL; QUAN, 2008).

Desse modo, tem-se que o SI adotado está alinhado aos processos empresariais da organização utilizada como unidade de análise. Assim, conclui-se que a Tecnologia da Informação tem potencial para oferecer suporte integral aos processos empresariais, incrementando, assim, a qualidade no processo principal do negócio e também nos processos de apoio da organização. Aspecto esse que pode ser corroborado por meio da literatura, pois Law e Jogaratnam (2005) consideram que a TI pode contribuir além das aplicações técnicas, podendo ser parte da abordagem de gestão para enfatizar a qualidade dos serviços no setor hoteleiro.

\subsection{Satisfação dos usuários com o Sistema de Informação adotado}

Este objetivo foi atingido por meio da aplicação de um questionário dividido em cinco agrupamentos de variáveis - constructos. O primeiro grupo trata de variáveis de perfil dos colaboradores. Em seguida, avalia-se a qualidade do sistema de informação adotado, qualidade de informação de saída, satisfação do usuário, uso real e impacto individual no colaborador.

Para a medição do nível de concordância com as afirmativas, foi utilizada uma escala do tipo Likert de sete pontos, onde a atribuição do número 1 representa uma característica negativa e o número 7, uma característica positiva inversa. Dessa maneira, foi estabelecido que os valores 1,2 e 3 correspondem a pontos desfavoráveis; os valores 4 e 5 pontos neutros; e os pontos favoráveis aqueles superiores a 5. Em seguida, são apresentadas a explicação e a representação das diferentes médias obtidas em cada um dos itens dos constructos avaliados através da média aritmética.

Todos os usuários do sistema de informação responderam o questionário, totalizando 20 usuários. O perfil dos respondentes quanto ao gênero foi: $55 \%$ pertencem ao sexo masculino e $45 \%$ ao sexo feminino. De acordo com os dados, os usuários estão alocados em cinco departamentos. 9\% estão alocados no Departamento de Alimentos e Bebidas (A\&B), 27\% trabalham no Departamento de Eventos e Convenções, 9\% no Departamento de Compras, 
46\% no Departamento de Hospedagem e mais 9\% no Departamento Financeiro. A respeito do tempo de empresa, observou-se que a maioria dos colaboradores estão na empresa há tempo superior a dois anos.

No que se refere ao constructo qualidade do sistema de informação adotado, tem-se que esse é composto por seis variáveis, as quais apresentaram médias que demonstraram a satisfação do usuário com o sistema de informação adotado: flexibilidade (cerca de $80 \%$ dos respondentes mostraram-se satisfeitos com a flexibilidade do SI adotado), capacidade de comunicação entre os módulos (cerca de 90\% dos respondentes mostraram-se satisfeitos com essa característica), capacidade de comunicação com outros sistemas de informação (aproximadamente $60 \%$ dos respondentes mostraram-se satisfeitos com esse aspecto do SI adotado), avaliação do tempo de resposta ( $85 \%$ das respostas apontaram satisfação com o tempo de resposta do SI), avaliação da capacidade de recuperação de erros (aproximadamente $75 \%$ dos respondentes sustentaram satisfação com a capacidade de recuperação do SI), avaliação de conveniência e avaliação de facilidade de interação (interface) (100\% dos usuários estão satisfeitos com esses aspectos). Dessa forma, os usuários mostraram-se satisfeitos com o SI adotado.

Quanto ao constructo qualidade de informação de saída, é composto pelas variáveis: volume, precisão, integridade, acuracidade, consistência, aceitação e formato das informações de saída. Os dados da pesquisa permitiram concluir que a qualidade de informação é satisfatória aos usuários, uma vez que as respostas apontaram $100 \%$ de satisfação para esses aspectos.

Os constructos satisfação do usuário, uso real e impacto individual no colaborador também obtiveram média considerada favorável. Salienta-se que mais de $80 \%$ dos colaboradores acreditam que a utilização do Sistema de Informação é essencial para a realização do trabalho. E cerca de $90 \%$ acreditam que a utilização de SI aumenta sua produtividade e eficácia. Resultado que também pode ser percebido na literatura vigente sobre o tema (WATKINS, 1995; LAW; JOGARATNAM, 2005).

Dessa forma, pode-se concluir que, sob a ótica dos usuários, o desempenho do sistema é satisfatório. Em todos os constructos avaliados, por meio da escala, foi observado que a maior concentração de frequência das respostas localizava-se na parte considerada favorável da Likert. Então, conforme investigação junto aos usuários, o sistema de informação está alinhado com os processos empresariais. 


\section{Conclusões}

A realização do presente estudo, que objetivou explorar as relações entre os processos empresariais e o sistema de informação adotado, proporcionou maior compreensão quanto ao relacionamento entre esses dois componentes da organização. Para tanto, como unidade de análise foi utilizada uma empresa do setor hoteleiro, justificável pela importância dessas organizações nos próximos anos e, sobretudo, da importância de que essas empresas estejam bem estruturadas e preparadas para prestar um serviço de excelente qualidade. Os objetos de análise foram os processos empresariais do hotel (aqueles que foram permitidos aos pesquisadores) e o os seis módulos, adquiridos e utilizados como suporte nas atividades, do sistema Desbravador, versão 3.0.

Quanto aos processos, a análise dos dados permitiu mapear 33 processos de negócio e também estruturar as atividades departamentais. A respeito da análise de processos, observaram-se diversos retrabalhos, o que pode levar a desperdícios de recursos. Nesse sentido, destaca-se o Departamento de Hospedagem - mais especificamente a área funcional Recepção - com retrabalho em muitos de seus processos. Com o acompanhamento dos processos empresariais, pode ser observado o suporte essencial que a tecnologia de informação adotada oferece aos processos. Há processos que despenderiam muito mais tempo, recursos e talvez algumas contratações para a empresa, se não existisse o sistema de informações. Dessa forma, pode ser identificada existência de uma estreita relação entre os processos empresariais e o sistema de informação adotado pela empresa. No que se refere ao alinhamento entre os processos empresariais e o Sistema de Informação adotado pela empresa, pode-se concluir, por meio do acompanhamento durante a pesquisa, que o SI oferece o suporte necessário aos processos da unidade de análise investigada, pois o sistema de informação oferece o suporte necessário aos processos. Mesmo assim, acredita-se que através do redesenho de alguns processos o SI adotado poderá ser mais bem empregado e, assim, promover maior alinhamento. Uma vez que, existem muitos processos em que há retrabalhos.

No que diz respeito às limitações desse estudo, tem-se que por motivos de segurança de informações relativas à competitividade, o Departamento de Recursos Humanos, que também age como auditor interno, não possibilitou acesso para mapeamento de processos nem para visualização dos módulos do sistema utilizado. Quanto às limitações metodológicas, pode-se 
citar o fato de não ter sido possível a realização de entrevistas, o que traria contribuições para uma melhor avaliação das variáveis.

Sugere-se, para estudos futuros, a atuação para um maior alinhamento na organização sobre eliminação dos retrabalhos mencionados nesta pesquisa, bem como realizar estudos em outras organizações do mesmo setor para que possam ser feitas comparações entre diferentes realidades empresariais, sendo possível obter uma ampla realidade do setor por meio do estudo de múltiplos casos. Ainda, pode-se realizar uma survey, visando à análise da satisfação de colaboradores de hotéis em relação ao sistema de informação adotado pelo respectivo empregador.

\section{Referências}

BARROS, A.I.S.; LEHFELD, N.A. S. Fundamentos de Metodologia Científica. $3^{\text {a }}$ edição. São Paulo: Pearson Makron Books, 2007.

BRASIL. Ministério do Turismo. Portal Turismo. Disponível em: http://www.turismo.gov.br/turismo. Acesso em: 03 set., 2011.

BRASIL. Competitividade no setor hoteleiro: Encontro entre MTur e hotelaria discute principais temas para fortalecer a atividade. Notícia. 2012. Disponível em: http://www.turismo.gov.br/turismo/noticias/todas noticias/20120810.html. Acesso em: 13 ago. 2012.

BROADBENT, M.; WEILL, P. Improving Business and Information Strategy Alignment: learning from the banking industry. IBM Systems Journal, vol. 32, n. 1, p. 162-179, 1993.

BRODBECK, A.F.; GALLINA, D.B. Um Modelo Aplicado de Gerenciamento de Processos de Negócio Alinhado aos Objetivos Estratégicos do Balanced Scorecard de uma Indústria Eletroeletrônica. In: Anais I Encontro de Administração da Informação. Florianópolis, 2007.

CAMISON, C. Strategic attitudes and information technologies in the hospitality business: an empirical analysis. International Journal of Hospitality Management, vol. 19, n. 2, p. 125-143, 2000.

CAMPOS, V.F. Controle da Qualidade Total. $8^{\text {a }}$ Edição. Rio de Janeiro: Block. 1999.

CARVELL, S.A.; QUAN, D.C. Exotic reservations - low prices guarantee. International Journal of Hospitality Management, vol. 27, n. 2, p. 162-169, 2008.

CARVALHO, L.F., ALVES, C.J.P. O Desafio da Infra-Estrutura Aeroportuária para a Copa do Mundo de 2014. XV Encontro de Iniciação Científica e Pós-Graduação do ITA (ENCITA), São Paulo, 2009.

CASTELLI, G. Administração hoteleira. 9a Edição. Caxias do Sul: EDUCS. 2003.

CAUCHICK MIGUEL, P.A. Metodologia de Pesquisa em Engenharia de Produção e Gestão de Operações. Rio de Janeiro: Elsevier. 2010.

COBANOGLU, C.; CORBACHI, K.; RYAN, B. A comparative study: the impact of technology in lodging properties in the United States and Turkey. International Journal of Hospitality Information Technology, vol. 2, n. 1, p. 23-40, 2001. 
COUTINHO, F.G.A.; CHAGAS, P.B. Instrumentos de operações de serviços para atendimento ao cliente utilizados por um hotel no interior do Estado do Paraná. In: Anais XXIX Semana do Administrador, Maringá, 2009.

DAVENPORT, T.H.; SHORT, J.E. The New Industrial Engineering: Information technology and Business Process Redesign. Sloan Management Review, vol. 31, n. 4, p. 1-31, 1990.

DAVIS, S.; DAVIDSON, B. 2020 Vision: Transfer your Business Today to Succeed in Tomorrow's Economy. New York: Simon \& Schuster. 1991.

DESBRAVADOR HOTEL AUTOMATION - Disponível em: http://www.desbravador.com.br/. Acesso em: 08 de agosto, 2012.

DOMINGUES, E.P., BETARELli JUNIOR, A.A., MAGAlHÃES, A.S. Copa do Mundo 2014: Impactos Econômicos no Brasil, em Minas Gerais e Belo Horizonte. Texto para discussão 382/2010. $29 \mathrm{p}$.

ESTEVÃO, C.M.S.; MAINARDES, E.W.; RAPOSO, M.L.B. Orientação para o Mercado no Segmento Hoteleiro: o caso português. Turismo em Análise, vol. 20, n. 3, p. 446-468, 2009.

FARRELL, C. An Onshore Play in Offshoring. Business Week New York, vol. 3991, n. 3, p. 90-91, 2005.

GIL, A. C. Como elaborar projetos de pesquisa. São Paulo: Atlas. 2010.

GOHR, C.F.; SANTOS, L.C. Estratégias Competitivas para Empresas Turísticas: um estudo no setor hoteleiro de um município do litoral catarinense. Turismo em Análise, vol. 21, n. 2, p. 297-319, 2010.

HAIR, J.F.P.J.; BABIN, B.; MONEY, A.H.; SOMOUEL, P. Fundamentos de métodos de pesquisa em administração. São Paulo: Bookman, 2006.

HENDERSON, J.C.; VENKATRAMAN, N. Strategic alignment: Leveraging information technology for transforming organizations. IBM System Journal, vol. 32, n. 1, p. 4-16, 1993.

HORNE, J. The Four 'Knows' of Sports Mega-Events. Leisure Studies, vol. 26, n. 1, p. 81-96, 2007.

IIVARI, J. An empirical test of Delone-Mclean of Information System Success. The Data Base for Advances in Information System, vol. 36, n. 5, p. 8-27, 2005.

INSTITUTO BRASILEIRO DE TURISMO (EMBRATUR). Disponível em: http://www.turismo.gov.br/turismo/o_ministerio/embratur/. Acesso em: 21 abr. 2012.

JAUHARI, V.; RISHI, M. Challenges faced by the hospitality industry in India: an introduction. Worldwide Hospitality and Tourism Themes, vol. 4, n. 2, p. 110-117, 2012.

JURAN, J.M. Planejando para a Qualidade. 3ª Edição. São Paulo: Pioneira. 1995.

KANDAMPULLY, J.; SUHARTANTO, D. The customer loyalty in the hotel industry: the role of customer satisfaction and image. International Journal of Contemporary Hospitality Management, vol. 12, n. 6, 346-351, 2000.

KAPLAN, R.S.; NORTON, D.P. A Estratégia em Ação: Balanced Scorecard. 10a Edição. Rio de Janeiro: Elsevier. 1997.

KAPLAN, R.S.; NORTON, D.P. Alinhamento. Rio de Janeiro: Elsevier. 2006.

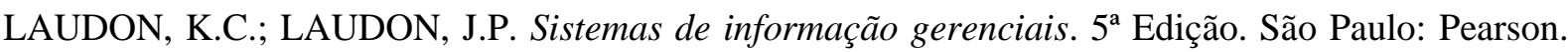
2004.

LAURINDO, F.J.B.; ROTONDARO, R.G. Gestão integrada de processos e da tecnologia da informação. São Paulo: Atlas. 2006. 
LAW, R.; JOGARATNAM, G. A study of hotel information technology applications. International Journal of Contemporary Hospitality Management, vol. 17, n. 2, p. 170-180, 2005.

LIMA FILHO, D.O.; MARCHIOTTI, I.Z.; SILVA, F.Q. Expectativas versus Satisfação dos Consumidores da Rede Hoteleira de Campo Grande-MS. Turismo em Análise, vol. 23, n. 1, p. 54-77, 2012.

LINDIC, J.; BALOH, P.; RIBIÈRE, V.M.; DESOUZA, K.C. Deploying information technologies for organizational innovation: Lessons from case studies. International Journal of Information Management, vol. 31, n. 2, p. 183-188, 2011.

LUFTMAN, J.N.; BULLEN, C.; LIAO, D.; NASH, E.; NEUMANN, C.; Managing the Information Technology Resource: Leadership in information Age. New Jersey: Pearson Prentice Hall. 2004.

LUFTMAN, J.N.; LEWIS, P.R.; OLDACH, S.H. Transforming the enterprise: The alignment of business and information technology strategies. IBM System Journal, vol. 32, n. 1, p. 198-220, 1993.

MAÇADA, A.C.; BORENSTEIN, D. Medindo a satisfação dos usuários de um sistema de apoio à decisão. In: Anais do XXIV Encontro Nacional dos Programas de Pós Graduação em Administração, Florianópolis, 2000.

MONZONI NETO, M.P.; OLIVEIRA, B.C.P.; KISS, B.C.K. Sustentabilidade e a Copa do Mundo de 2014: Desafios e Oportunidades na Gestão das Emissões de Gases de Efeito Estufa. Simpósio de Administração da Produção, Logística e Operações Internacionais. Anais SIMPOI 2011. São Paulo.

NEVES, I.A.; SEMPREBOM, E.; LIMA, A.A. COPA 2014: Expectativa e Receptividade os Setores Hoteleiro, Gastronômico e Turístico na Cidade de Curitiba. Simpósio de Administração da Produção, Logística e Operações Internacionais. Anais SIMPOI 2011. São Paulo. 2011.

NOLAN, R.L. Managing the Crises in Data Processing. Harvard Business Review, vol. 57, n. 2, p. 115-126, 1979.

O’BRIEN, J.A. Sistemas de Informação e as decisões gerenciais na era da internet. $3^{\text {a }}$ Edição. Rio de Janeiro: Saraiva. 2010.

OHMANN, S.; JONES, I.; WILKES, K. The Perceived Social Impacts of the 2006 Football World Cup on Munich Residents. Journal of Sport and Tourism, vol. 11, n. 2, p. 129-152, 2006.

PORTER, M.E.; MILLAR, V.E. Como a informação proporciona vantagem competitiva. In: PORTER, M.E. Competição - on competition: estratégias competitivas essenciais. Rio de Janeiro: Campus, 1999.

RAMOS, A.S.M.; RODRIGUES, P.A.B. Tecnologia de Informação: Utilização e Vantagem Competitiva no Hotel Pestana Natal. In: Anais do XIII Simpósio de Engenharia de Produção, Bauru, 2006.

REZENDE, D.A.; ABREU, A.F. Tecnologia da informação aplicada a sistemas de informação empresariais: o papel estratégico da informação e dos sistemas de informação nas empresas. $3^{\text {a }}$ Edição. São Paulo: Atlas. 2003.

RICHARDSON, R. J. Pesquisa Social: métodos e técnicas. $3^{\text {a }}$ Edição. São Paulo: Atlas. 1999.

ROCKART, J.F.; EARL, M.; ROOS J. Eight Imperatives for the New IT Organization. Sloan Management Review, vol. 38, n. 1, p. 43-55, 1996.

ROSEN, K.T.; HOWARD, A.L. E-retail: gold rush or fool's gold? California Management Review, vol. 42, n. 3, p. 72-100, 2000. 
SABHERWAL, R.; CHAN, Y.E. Alignment between business and IS strategies: a study of prospectors, analyzers and defenders. Information Systems Research, vol. 12, n. 1, p. 1-33, 2001.

SANTOS, C.S. Introdução à gestão pública. São Paulo: Saraiva. 2006.

SANTOS, M.R. O Futebol na Agenda do Governo Lula: um salto de modernização (convencional) rumo a Copa do Mundo FIFA 2014. Dissertação de Mestrado (Programa de Pós-Graduação de Educação Física). Universidade de Brasília. 2011.

SEIXAS, T., LOPES, J.P.S.R. COPA DO MUNDO 2014: Um Estudo sobre o Processo de Candidatura de Pernambuco. Revista Intercontinental de Gestão Desportiva, vol. 2, n. 1, p. 1-15, 2012.

SHETH, J.N.; ESHGHI, A.; KRISHNAN, B.C. Marketing na Internet. Porto Alegre: Bookman. 2002.

SIGUAW, J.; ENZ, C.A. Best practices in information technology. Cornell Hotel and Restaurant Administration Quarterly, vol. 40, n. 5, p. 58-71, 1999.

VAN HOOF, H.B.; VERTEETEN, M.J.; COMBRINK, T.E. Information technology revisited: international lodging-industry technology needs and perceptions: a comparative study. Cornell Hotel and Restaurant Administration Quarterly, vol. 37, n. 6, p. 86-91, 1996.

TEIXEIRA, R.M. Competências e Aprendizagem de Empreendedores/Gestores de Pequenas Empresas no Setor Hoteleiro. Turismo em Análise, vol. 22, n. 1, p. 195-219, 2011.

TURBAN, E.; MCLEAN, E.; WETHERBE, J. Information Technology for Management: making connection for strategic advantage. John Wiley \& Sons, Inc.. 1999.

TURBAN, E.; RAINER Jr., R.K.; POTTER R.E. Introdução a sistemas de informação: uma abordagem gerencial. Rio de Janeiro: Elsevier. 2007.

WARD, J. PEPPARD, J. Strategic planning for information systems. $3^{\text {a }}$ Edição. New York: John Wiley \& Sons, Inc. 2002.

WATKINS, E. High tech meets high touch. Lodging Hospitality, June, p. 28, 1995.

WEILL, P.; VITALE, M. Assessing the Health of an Information Systems Application Portfolio: An Example from Process Manufacturing. MIS Quarterly, vol. 23, n. 4, p. 601-624, 1999.

YELKUR, R.; DaCOSTA, M.M.N. Differential pricing and segmentation on the Internet: the case of hotels. Management Decision, vol. 39, n. 4, p. 252-262, 2001.

\section{Recebido em: 17/08/2012 (1 ${ }^{\text {a }}$ versão) $09 / 06 / 2013$ (última versão)}

Aprovado em: 18/02/2014 